\title{
Le patrimoine en France : état des lieux, historique et perspectives
}

\begin{abstract}
$\grave{A}$ la suite des crises financières et économiques récentes, exceptionnelles par leur caractère aigu, le débat social a eu tendance à se polariser sur les incertitudes qui pesaient déjà depuis quelques années sur l'avenir socioéconomique de la France et sur leurs conséquences en matière de niveau de vie, de consommation et d'épargne.
\end{abstract}

Le chômage est reparti à la hausse. Les dépenses de santé vont peser de plus en plus lourd dans le budget des ménages, notamment en raison du coût de la dépendance. Les jeunes générations voient leur avenir avec appréhension du fait de l'accroissement de la dette publique, des difficultés d'insertion sur le marché du travail et de la dévalorisation des diplômes. Le système de retraite, dont l'équilibre à long terme n'est guère assuré aux regards des évolutions contrastées de la durée de vie active et de l'espérance de vie, devrait connaître des réformes profondes (Arrondel et Masson, 2007a).

Ces perspectives, inquiétantes sous nombre d'aspects, ont ainsi ranimé le débat politique entre les défenseurs de l'État providence, tel qu'on l'a connu depuis la fin de la deuxième guerre mondiale, et les tenants d'un système sollicitant de plus en plus l'initiative individuelle par l'intermédiaire de l'épargne privée (Masson, 2009). L'accumulation patrimoniale pour les vieux jours serait-elle la réponse aux incertitudes portant sur le niveau des pensions ou sur les dépenses de santé des personnes âgées ? En toile de fond, c'est aussi le problème de l'équité intergénérationnelle qui est soulevé, un thème abordé d'abord par les économistes nord-américains (Kotlikoff, 1992), puis repris par des chercheurs français, économistes et sociologues (en particulier Chauvel, 2002).

La crise des subprimes et ses conséquences dans le monde de la finance et de la banque ont mis en avant les aspects " prise de risque » qui sont au cœur de toute décision d'affectation de la richesse accumulée entre les divers produits disponibles, avec l'omniprésent arbitrage entre l'espérance du rendement et sa variabilité. Les feux de l'actualité ont ainsi contribué à relancer l'intérêt pour l'économie et la sociologie de l'épargne et du patrimoine mais ils ont ainsi fait ressortir à quel point la connaissance de ces sujets est encore partielle.

Trancher, par exemple, le débat renouvelé sur l'efficience de l'État providence sous sa forme actuelle supposerait pour être convenablement documenté, que l'on sache répondre à toute une série de questions. Par exemple, les Français se protègent-ils suffisamment contre les aléas de la vie et ceux qui sont endettés pourront-ils faire face à leurs remboursement en cette période de crise économique ? Préparent-ils leurs vieux jours raisonnablement tôt ou faut-il chercher à les éduquer financièrement pour les aider à mieux les préparer? Doit-on les inciter fiscalement à plus de solidarités familiales? Les solutions mises en place, tournant autour de l'épargne privée et des arbitrages intergénérationnels, sont-elles capables de survivre à une crise financière (effondrement des cours boursiers, tensions sur le marché immobilier)?

\section{L'émergence du patrimoine dans la pensée économique}

$\mathbf{E}$ n matière de patrimoine, la crise récente a contribué à faire ressurgir au premier plan des interrogations que l'on avait un peu hâtivement considérées comme caduques ou résolues en période de croissance continue. D'autres questions, pour lesquelles l'intérêt n'avait pas dimi- 
nué, n'ont pas encore reçu de réponse claire, bien qu'elles semblent cruciales pour l'avenir de nos sociétés. Les difficultés sont à la fois théoriques et statistiques et les défis apparaissent redoutables pour un domaine d'analyse relativement récent, comme le montre un bref détour historique.

\section{Le patrimoine, grandeur négligée des modèles macroéconomiques}

La macroéconomie a sans doute été la première à mettre en exergue l'importance, pour l'équilibre et le développement économique, du comportement d'épargne des ménages, ou plutôt du partage du revenu entre épargne et consommation : un excès chronique d'épargne ne risque-t-il pas de mettre en péril l'existence d'un équilibre ou un rythme suffisant de croissance à long terme ? Ou encore, une relance par la consommation est-elle souhaitable ? Ces questions tournant autour du rôle de l'épargne ont toujours eu une place centrale dans la réflexion macroéconomique. Le patrimoine constitué par l'épargne occupe, en revanche, une place plus discrète, sans doute parce qu'il s'agit d'une variable de stock. Il est traité davantage comme un résidu que comme une grandeur désirée pour elle-même. Tout au plus lui attribue-t-on quelques effets de second ordre dont le phénomène dit «d'encaisses réelles ».

Les modèles macroéconomiques des années 1970 sont bien dans cette lignée : ils comportent tous une fonction de consommation, mais pas d'équation de patrimoine. En outre, la macroéconomie traditionnelle ne fournit pas un cadre intégré pour l'étude des comportements du consommateur : les approches théoriques des concepts standard d'épargne de précaution, de transaction ou de spéculation sont diverses, faute sans doute de tests empiriques suffisamment précis pour les départager. Les développements plus récents introduisent certes de nouveaux aspects affinant l'analyse des comportements (les contraintes de liquidité en particulier), mais il apparait clairement que les progrès de la connaissance des phénomènes macroéconomiques passent aujourd'hui par une meilleure compréhension des comportements microéconomiques, elle-même conditionnée par l'enrichissement des données disponibles.

\section{L'approche microéconomique du patrimoine : motifs d'accumulation et inégalités}

Inspirée au départ par les questions posées par les macro-économistes, la microéconomie du consommateur s'est longtemps focalisée sur les comportements de consommation : simple reste, l'épargne n'était pas dotée d'utilité propre. Une première étape est franchie avec les modèles dits « de cycle de vie ». Mais dans les versions de base, en l'absence d'incertitude et de transferts intergénérationnels, l'épargne sert encore uniquement de vecteur au transfert du pouvoir d'achat d'une période à l'autre, pour adapter le profil inter-temporel des ressources à celui de la consommation désirée ; le patrimoine, stock d'épargne accumulée, n'est qu'une réserve de consommation différée ayant vocation à disparaître en fin de vie (Modigliani et Brumberg, 1954).

Les modèles de cycle de vie ont été amenés à se diversifier, les variantes les plus simples étant incapables de rendre compte des phénomènes observés. Nécessairement posé dans un cadre intertemporel, le comportement d'épargne se doit d'aborder les problèmes liés à la prise en compte du caractère fondamentalement incertain de l'avenir et donc doit reposer sur une modélisation du consommateur en présence d'incertitude. Établir la simple rationalité de l'agent dans ce contexte est encore sujet à débat, chaque « axiome » (les plus connus étant ceux de l'utilité espérée selon Von Neumann-Morgenstern) étant systématiquement en contradiction avec les comportements de fractions non négligeables de la population. D'autres extensions ont concerné l'horizon décisionnel des agents, élargi à la descendance proche ou, au-delà, jusqu'à un comportement dynastique (modèles à la Barro). Et il a été reconnu que l'individu n'était pas qu'un simple épargnant mais 
pouvait également investir au plan éducatif et professionnel pour améliorer ses ressources. Toute une frange de la microéconomie insiste désormais sur l'accumulation du capital non matériel - le « capital humain » selon Gary Becker (1991) - même si elle a peu tenu compte de l'hétérogénéité de ces capitaux non humains comme le fera la sociologie de Pierre Bourdieu, distinguant capital affectif, symbolique, idéologique, culturel, social, et enfin économique.

Parallèlement à ces développements concernant la modélisation des comportements, on a assisté à un renouvellement de la problématique économique de l'inégalité patrimoniale, au départ centrée sur le seul rôle de l'héritage comme vecteur privilégié de la reproduction intergénérationnelle des fortunes : les riches sont-ils fils de riches? L'enjeu s'est porté sur le pouvoir explicatif des modèles de cycle de vie, éventuellement élargis aux transmissions. En prenant en compte en plus de l'héritage de nouvelles variables, tels que l'âge, le revenu permanent, les imperfections des marchés de l'assurance et du crédit, etc., ces modèles cherchent à rendre compte de la répartition des patrimoines au sein des ménages, du moins hors les $1 \%$ les plus riches. Il a fallu en effet attendre ces dernières années pour que l'on tente d'expliquer le degré élevé de la concentration des patrimoines au sein des ménages les plus fortunés, à partir notamment de l'incertitude des revenus du capital.

Les recherches empiriques n'ont pas suivi le même cours en France et outre-Atlantique. En France, les approches tant économiques que sociologiques de l'inégalité se sont davantage focalisées sur le bas de la distribution des revenus que sur la concentration des richesses. La pauvreté a monopolisé une grande partie de l'attention de sorte que l'on connaît mieux désormais les mécanismes générateurs d'exclusion sociale que ceux qui sont à l'origine des grandes fortunes. Si l'on excepte quelques travaux sur la répartition du patrimoine, réalisés entre autres par les auteurs de cet article, l'intérêt ne s'est déplacé vers le haut de la distribution que récemment, à partir de l'étude historique de Piketty (2001) qui porte seulement sur les revenus. Mais, alors qu'aux États-Unis le thème de la polarisation de la société est récurrent depuis plusieurs années (le phénomène de la « shrinking middle class »), seules quelques études pionnières ont été réalisées sur le sujet pour la France (1). Ce sera sans doute un enjeu de la recherche pour les années à venir que de renouveler les mesures de façon à documenter les effets de la crise récente sur l'homogénéité de la structure sociale et vérifier que le «strobiloïde » de la pyramide des revenus ou des patrimoines, cher à Louis Chauvel, ne s'est pas transformé en structure bipolaire.

\section{Le patrimoine des français : controverses d'hier et d'aujourd'hui}

L'économie de l'épargne et des patrimoines apparait comme ayant toujours été marquée par l'abondance des questions ouvertes. En 1996, dans la préface du premier numéro spécial d'Économie et Statistique consacré à l'étude du patrimoine des Français, Masson et Verger identifiaient une série de problématiques essentielles pour faire progresser la connaissance. Quelles parts des inégalités de patrimoine sont expliquées respectivement par les inégalités de revenus, les héritages et l'hétérogénéité des préférences des ménages (degré de préférence pour le présent ou d'aversion au risque)? Quels sont les liens entre les motifs d'accumulation et la composition des patrimoines ? Quelle est l'évolution à long terme des inégalités de fortunes, et les différents pays industrialisés se ressemblent-ils sur ce point ? Quelles spécificités peut-on discerner dans le comportement d'accumulation et la composition des patrimoines des indépendants ? Deux autres points concernaient la famille : le patrimoine est-il affaire de couple ou de lignée ? L'équirépartition entre héritiers est-elle respectée dans l'ensemble des transferts intergénérationnels ? (Masson et Verger, 1996).

1. Echevin et Parent (2002), malgré le développement d'outils statistiques adaptés (indices de Foster-Wolfson ou d'Esteban-Ray ) n'ont pas vraiment fait école. Il est vrai que leurs résultats, assez difficiles à interpréter, n'ont pas mis en évidence, au cours des années 1980 et 1990, des évolutions d'ampleur comparable à celles qui ont été observées aux États-Unis. 
D'autres questions, parfois plus ponctuelles, abondent actuellement dans la littérature. Elles se présentent la plupart du temps sous forme d'énigmes (puzzles) qui témoignent des écarts entre les modèles, même les plus avancés, et les faits observés. Parmi les «puzzles » les plus connus, on peut citer «l'énigme de la prime de risque » (pourquoi les ménages détiennent-ils si peu d'actions ?) ; la désaffection pour la rente viagère en dépit des caractéristiques séduisantes de ce placement ; ou encore la grande diversité des ratios patrimoine/revenu à la veille de la retraite. D'autres interrogations concernent plus spécifiquement la gestion du portefeuille financier. Pourquoi les ménages privilégient-ils les actions nationales ? Pourquoi les investisseurs réagissent-ils de façon dissymétrique aux gains et aux pertes, etc. ?

Le nombre de débats en suspens s'explique certes par la complexité des comportements en jeu, auxquels on prête souvent une rationalité excessive dans un contexte temporel où l'incertitude est omniprésente (Arrondel et al., 2004), mais aussi par la relative jeunesse des dispositifs d'observation statistique.

\section{La connaissance statistique est-elle à la hauteur des enjeux ?}

$\mathbf{F}$ ace à l'importance de ces enjeux, l'information statistique est encore en retrait même si des progrès notables ont été accomplis depuis une vingtaine d'années. La collecte réalisée au moyen d'enquêtes par sondage aléatoire remonte à l'immédiat après-guerre et le dispositif qui s'est alors mis progressivement en place était ciblé sur les problèmes d'une économie en reconstruction.

\section{Les progrès lents de la collecte}

Après le recensement de 1946, les enquêtes auprès des ménages se développent dans le sillage des premières opérations auprès des entreprises : les départs en vacances sont étudiés en 1949, l'emploi est suivi régulièrement à partir de 1950, et 1951 voit l'apparition de la première enquête généraliste sur la consommation sous une forme proche des enquêtes Budgets de famille qui, depuis, n'ont jamais cessé d'être réalisées, à un rythme approximativement quinquennal, etc. Ont suivi des enquêtes sur les loyers, l'écoute de la radio, l'habillement, les biens durables et l'ameublement, l'utilisation de l'énergie, les intentions d'achat, la consommation alimentaire, la santé, les caractéristiques du logement, les emplois du temps, la formation et la qualification professionnelle.

Mais le revenu (a fortiori, le patrimoine) était alors considéré comme un domaine presque « tabou », quasi-impossible à étudier au moyen d'une enquête. E. Malinvaud, exploitant une des premières enquêtes Habillement, avait même écrit «le lecteur regrettera certainement de voir qu'aucune question sur le revenu n'a été retenue dans le questionnaire... Toute tentative de description statistique du niveau de revenus auprès d'un échantillon aléatoire d'individus est vouée à un échec certain » (Bertaud et Malinvaud, 1953). À cette époque, c'est par le truchement de la catégorie sociale que la position économique du ménage était appréhendée.

Ce n'est que, timidement, dans la seconde moitié de la décennie soixante, puis plus fréquemment dans les années 1970 que la statistique publique a osé aborder les dimensions pécuniaires dans les enquêtes, d'abord avec l'évaluation des revenus qui s'est progressivement généralisée, puis avec des essais de mesure de l'épargne et des comportements d'accumulation patrimoniale. Le premier « pilote » a été réalisé en 1965, la première tentative d'enquête en 1967, mais c'est seulement en 1973 qu'a été lancée une enquête Épargne ambitieuse, avec l'idée de mesurer l'épargne comme une variation du stock de patrimoine entre deux années (ce qui nécessitait deux 
interviews du même ménage). Deux cycles d'enquêtes ont ainsi été réalisés (1973-1975 et 19741976) selon un protocole qui prévoyait également des appariements avec des fichiers bancaires, afin de valider les mesures de montant obtenues. L'expérience a été interrompue car les techniques d'exploitation alors disponibles n'avaient pas permis d'obtenir des résultats exploitables : les évolutions obtenues par différence étaient généralement incompatibles avec celles décrites spontanément par l'enquêté dans la partie rétrospective du questionnaire. Et l'appariement avec les données bancaires, dont les résultats n'ont jamais été publiés, diagnostiquait des sous-estimations de montant importantes, qui plus est variables selon les produits mais aussi selon les catégories de répondants (par exemple selon la catégorie sociale).

Pendant près de dix ans, la statistique publique n'a plus abordé, par enquête, le sujet du patrimoine, laissant ce soin au Crep (Centre de Recherche Économique sur l'Épargne) qui a réalisé diverses enquêtes entre 1975 et 1980, en rencontrant d'ailleurs des difficultés de même nature.

Dans la préface du numéro spécial d'Économie et Statistique consacré au patrimoine en 2005, S. Lollivier a retracé l'histoire de ces balbutiements de la collecte statistique du patrimoine (Lollivier, 2005).

En 1986, l'enquête Actifs financiers conduite par l'Insee n'avait abordé que très prudemment les questions de montants, renonçant au recueil d'estimations détaillées produit par produit au profit d'une seule question sur le montant total du patrimoine, posée sous forme de tranches assez larges, et peu informative sur le haut de la distribution : la limite de la dernière tranche avait volontairement été choisie inférieure au seuil d'imposition sur la fortune pour éviter que les ménages ne suspectent une opération de contrôle fiscal.

L'Institut s'est ensuite efforcé de répondre à la demande sociale pressante sur les aspects quantitatifs du domaine. Une alternance avait alors été prévue entre des enquêtes complexes réalisées à un rythme quinquennal et des opérations plus légères effectuées au milieu de chaque période, pour évaluer les évolutions conjoncturelles des taux de détention.

Le nombre de sujets à aborder étant important - montants détenus sur tous les actifs, financiers et immobiliers (avec une attention particulière portée sur les actifs à usage professionnel) ; description du passif (endettement) ; carrières patrimoniales (en distinguant l'accumulation personnelle des transferts reçus ou effectués) - il avait alors été décidé de n'approfondir qu'une fois sur deux (soit tous les dix ans) les transmissions intergénérationnelles et l'endettement, en établissant un roulement entre ces deux thèmes. Ce dispositif n'a pas réellement été appliqué : des enquêtes Détention d'actifs ont été réalisées dans le cadre des enquêtes permanentes sur les conditions de vie (EPCV) en 1996 et en 2000. Elles ont été abandonnées depuis parce qu'elles ne renseignaient pas sur les montants.

Les tentatives d'alléger les enquêtes Patrimoine elles-mêmes, qui répondaient au souci de réduire la charge pesant sur les ménages tout en diminuant les coûts, se sont soldées par un semi-échec, avec une perte sensible de la richesse de l'information collectée et corrélativement des possibilités d'exploitation réduites. Le stock de patrimoine et le flux d'épargne restent de fait des variables difficiles à observer. Les problèmes de mémoire, d'identification des actifs financiers, toujours plus complexes dans leurs caractéristiques, de valorisation des produits en dehors de toute transaction, posent de redoutables défis au statisticien qui ne saurait s'accommoder d'une collecte sommaire et rapide. L'enquête Patrimoine 2004, qui fournit une part importante du matériau statistique utilisé dans les articles du présent dossier, est revenue à un questionnement plus complet. En dépit de leurs imperfections, les données obtenues constituent ainsi une base solide, et ce d'autant plus que les estimations des grands postes du patrimoine ont fait l'objet de traitements statistiques d'apurement complexes, sujet traité dans l'article de Eric Gautier et Cédric Houdré. 
À partir des données brutes, la plupart du temps collectées sous forme de tranches assez larges et parfois incomplètes (2), les deux auteurs ont mobilisé toutes les ressources de la recherche statistique en matière d'imputation et de correction de la non-réponse pour fournir un fichier de montants estimés de la meilleure qualité possible.

Pour éviter toute ambiguïté, il faut souligner que l'article ne rend compte que de la première étape des travaux statistiques effectués sur les données, celle qui se borne à considérer l'enquête elle-même sans la rapprocher de données externes : les questions liées au calage sur les données de comptabilité nationale n'y sont pas abordées. Le caractère technique de l'article nécessitera sans aucun doute un effort de la part du lecteur, mais un effort qu'il est indispensable de fournir si l'on veut pouvoir aborder les statistiques sur le sujet avec ce qu'il faut d'esprit critique, en étant capable de faire le tri entre ce qui est établi de matière certaine et ce qui reste soumis à conjecture.

Que le bilan relatif à l'état de la connaissance soit en demi-teinte ne doit pas occulter les réels progrès de la connaissance dans le domaine : même s'il reste des questions en suspens, il ne fait aucun doute que l'on sait désormais beaucoup de choses sur le patrimoine et l'épargne. Cette connaissance du paysage patrimonial français, disséminée dans de très nombreuses publications récentes, émanant tant des statisticiens de l'Insee que des chercheurs, constitue une sorte de prérequis pour aborder les articles du présent dossier.

\section{Épargne et patrimoine : un état des lieux des données nationales}

En France, depuis le début des années 1990, le taux d'épargne financière se situe en termes de flux, avec les normes actuelles de la comptabilité nationale, au-dessus de $5 \%$ du revenu disponible et le taux d'épargne global oscille autour de $15 \%$. La France est ainsi parmi les pays les mieux placés au sein de l'Europe, avec l'Allemagne et l'Italie.

En termes de stock, fin 2007 et selon la comptabilité nationale, le patrimoine (brut) des ménages représentait près de 10550 milliards d'euros, soit une augmentation de $144 \%$ en dix ans. Ce stock de patrimoine représente environ 5,5 fois le PIB français. L'encours des dettes se situant autour de 1150 milliards d'euros, le patrimoine net s'élève à 9400 milliards d'euros. L'endettement des ménages français au cours des dix dernières années est ainsi passé de $41 \%$ à $61 \%$ du PIB.

Rapporter ces masses au revenu disponible des ménages de l'année est encore plus parlant : le stock de patrimoine représentait 4,5 fois le revenu en 1997 et 7,5 en 2007 ; l'endettement est passé quant à lui de $64 \%$ du revenu à $95 \%$.

La crise financière actuelle a provoqué des inquiétudes quant à ces niveaux d'endettement des ménages qui sont très variables dans les pays européens, la moyenne européenne se situant aux alentours de $90 \%$ du PIB (contre près de $140 \%$ aux États-Unis) : ainsi en 2004 pour les pays les plus endettés, les encours de dettes représentaient plus de $200 \%$ du PIB aux Pays-Bas et près de $130 \%$ au Royaume-Uni ; parmi les moins endettés, l'Italie présentait une dette se montant à $37 \%$ du PIB.

Au cours des dernières années, l'évolution de la richesse résulte essentiellement de la valorisation du patrimoine immobilier. Ainsi, sur la période 1997-2004 précédant l'enquête Patrimoine (Girardot et Marionnet, 2007), l'augmentation du patrimoine global de $64 \%$ s'expliquait principalement par celle du patrimoine immobilier, loin devant celle du patrimoine financier. Plus

2. Les déclarations de montant, même en tranches, présentaient encore des incohérences internes, entre les montants détaillés par produit et les valeurs globales du patrimoine par exemple. 
des trois quarts de l'augmentation de la richesse totale pouvait ainsi s'expliquer par celle de l'immobilier, variation dont la composante prix $(+61 \%)$ était nettement supérieure à la composante volume $(+17 \%)$. Inversement, le quart restant, dû à la variation du patrimoine financier, s'expliquait essentiellement par des acquisitions nouvelles, et non par les fluctuations des prix sur le marché des capitaux (3).

Cette importance des effets prix doit nous conduire à relativiser ce qui, à première vue, pourrait sembler un constat univoque de l'amélioration de la situation des ménages. Ce qui est sans aucun doute vrai pour un propriétaire de logement en position de vendeur, l'est beaucoup moins dans d'autres situations (abaissement de fait de la limite d'imposition à l'ISF, renchérissement des transmissions patrimoniales intergénérationnelles...).

Parallèlement, la structure des patrimoines français s'est profondément modifiée : en 1997, 58 \% du patrimoine total était constitué d'immobilier alors que sept ans plus tard cette proportion était de deux tiers (Boissinot et Friez, 2006). Les évolutions plus récentes n'ont pas modifié cette répartition.

\section{Que nous dit l'enquête « Patrimoine 2004 » sur la richesse en France ?}

$\mathbf{L}$ es montants que déclarent les ménages, quand on leur demande la valeur globale de tout ce qu'ils possèdent, sont inférieurs à ceux de la comptabilité nationale (Arrondel et al., 1996, Girardot et Marionnet, 2007). Globalement, les enquêtes recensent environ 70 à $75 \%$ du patrimoine de la comptabilité nationale (moins de $40 \%$ pour le portefeuille financier). Des entretiens qualitatifs menés auprès de ménages interviewés (Cohen et Demmer, 2004) révèlent par exemple que certains d'entre eux, interrogés sur la valeur globale de leur patrimoine, ont eu tendance à ne compter que les actifs explicitement recensés dans le questionnaire et n'ont donc pas respecté la consigne donnée de compter toutes les possessions susceptibles d'être vendues (incluant biens d'équipement, biens précieux, œuvres d'art, stock-options, etc.). Par ailleurs, les chiffres déclarés au moment d'évaluer le portefeuille « titre », diffèrent selon que les épargnants donnent la valeur d'achat ou la valeur du jour. Il est pourtant précisé que les montants recherchés sont ceux aux cours du jour, correspondant à la valeur actuelle du patrimoine, mais c'est une consigne difficile à faire respecter, soit par méconnaissance du ménage (4), soit pour d'autres raisons plus profondes, comme le refus de tenir compte des plus (ou moins)-values tant qu'elles ne sont pas réalisées. Il est en effet assez surprenant que la consigne soit violée dans un aussi grand nombre de cas. En ce qui concerne les assurances, $45 \%$ des détenteurs répondent en donnant une valeur d'achat ; pour les actions, la proportion, plus faible, atteint néanmoins $30 \%$ (comme pour l'épargne salariale). Nul n'est donc besoin de souligner à quel point, dans une période de croissance des indices boursiers, cela peut créer des divergences avec les modes d'évaluation de la comptabilité nationale, qui, elle, repose strictement sur les cours du jour.

L'enquête Patrimoine 2004 de l'Insee situe, en métropole, le patrimoine moyen (brut) des Français autour de 170000 euros (chiffres non calés sur ceux de la comptabilité nationale), et le patrimoine médian autour de 100000 euros (Cordier et al., 2006). Les $10 \%$ des ménages les plus riches (ceux dont la fortune excédait 382000 euros) détenaient environ $45 \%$ du total du patrimoine français.

3. En 2004 , le marché boursier, après l'explosion de la bulle internet, venait juste de repartir à la hausse (depuis le premier trimestre 2003).

4. Cette méconnaissance a différentes origines : le ménage laisse la gestion de son portefeuille financier à son banquier et ne se tient pas au courant des évolutions de cours; ou il n'a pas connaissance de transactions dans son entourage proche qui lui permettent d'évaluer avec un minimum de précision la valeur de ses résidences habituelles; de plus, cette évaluation est rendue particulièrement difficile quand la propriété est répartie entre plusieurs personnes - phénomènes d'indivision, d'usufruit. 
À titre de comparaison, l'enquête Share (Christelis et al., 2005), qui mesure les patrimoines avec une méthodologie comparable (5) en Europe pour les plus de 50 ans situe, au début des années 2000, la fortune médiane des Français d'âges mûrs entre 120000 et 140000 euros (nets), devant l'Europe du Nord, l'Allemagne (moins de 100000 euros), l'Autriche et la Grèce (entre 100 et 120000 euros) mais derrière l'Europe du Sud (plus de 140000 euros en Espagne et en Italie). La réalité patrimoniale est donc différente selon les pays d'Europe.

\section{Les inégalités}

Sur des données couvrant le XIX ${ }^{\mathrm{e}}$ et le $\mathrm{XX}^{\mathrm{e}}$ siècle, Piketty et al. (2006) ont mis en évidence que les inégalités de patrimoines ont crû jusqu'en 1914, pour décroître ensuite tout au long du $\mathrm{XX}^{\mathrm{e}}$ siècle. Mais Landais (2007) montre que les inégalités de revenus auraient augmenté au cours de la dernière décennie en raison de la forte croissance des revenus du patrimoine. Les inégalités de patrimoine auraient-elles alors connu la même tendance?

Il est difficile d'étudier précisément la distribution des fortunes à partir des enquêtes Patrimoine de l'Insee car celles-ci se veulent représentatives de la population française dans son ensemble et l'échantillon n'est pas assez ciblé en direction des ménages les plus riches. Certes une surreprésentation des ménages aisés est adoptée, mais insuffisante sans doute pour une bonne observation des plus hautes fortunes (6). Quoiqu'il en soit, l'inégalité des fortunes serait restée globalement stable entre 1992 et 2004 (Cordier et al., 2006), avec cependant une hausse tendancielle en fin de période (Girardot et Marionnet, 2007).

Le patrimoine moyen des plus de 50 ans est plus élevé en 2004 qu'en 1992, alors que la tendance est inverse pour les moins de 30 ans (Cordier et al., 2006). En outre, les inégalités de patrimoine se sont accrues au sein des ménages aux revenus les plus modestes (Cordier et al., 2006), ou entre les propriétaires et les non-propriétaires de leur logement principal.

En résumé, même s'il est difficile de mettre en évidence une augmentation globale nette des inégalités de richesse, certaines catégories, souvent les plus jeunes et les plus démunies, ont vu leur patrimoine croître moins rapidement que d'autres, sinon même stagner.

Ce constat est toutefois à interpréter avec précaution, puisque « âge biologique » et « âge social» ont divergé au cours de la période récente avec un retard systématique de tous les calendriers : âge au premier emploi, âge de départ du foyer parental, âge à la première union stable, âge à la première naissance, âge à l'achat d'un logement etc. À 19 ans aujourd'hui, on est ainsi plus « jeune » qu'à 19 ans hier. En conséquence, avoir moins de patrimoine à cet âge aujourd'hui atténue le caractère inquiétant du constat. En revanche, le fait que les écarts entre les âges soient plus importants en France qu'ailleurs (cf. infra) pourrait l'être davantage (7). Un autre élément qui complique l'analyse des évolutions des inégalités patrimoniales sur la période récente vient de ce que le patrimoine est habituellement une « affaire de ménages » et non d'individus (8). Or, la stabilité temporelle des ménages s'est progressivement réduite : les séparations et divorces,

\footnotetext{
5. Il s'agit de montants nets de l'endettement et sans valorisation des droits à retraite accumulés dans le cas d'existence de régimes de retraite par répartition.

6. Pour cela, des études complémentaires à partir d'autres sources, comme l'Impôt de Solidarité sur la Fortune (ISF) ou les enquêtes Mutations à Titre gratuit de la DGFIP, sont nécessaires, bien que ces données elles-mêmes ne permettent pas toujours une mesure fine des évolutions récentes (les sources fiscales ne sont disponibles, dans leur version définitive apurée, qu'après un délai de plusieurs années).

7. La difficulté d'interprétation des inégalités de richesse selon l'âge avait déjà été soulignée au début des années 1980 par A. Babeau : le fait que, sur la période antérieure, l'écart entre les patrimoines moyens des ménages jeunes et plus âgés avait doublé ne devait pas forcément être interprété comme une augmentation de l'inégalité : au début de la période incriminée, les jeunes sans patrimoine étaient en effet des personnes sans formation, qui n'auraient jamais la possibilité d'acquérir du patrimoine ; en fin de période, il s'agissait au contraire de personnes poursuivant des études longues et donc appelées à s'enrichir. Seulement, la dévalorisation constante des diplômes au cours des années 1990 rendrait cette interprétation optimiste moins pertinente pour les années récentes...

8. Les contrats de mariage en séparation de biens ont toujours été minoritaires (Barthez et Laferrère, 1996).
} 
générateurs de partage des patrimoines dans des conditions de marché souvent peu favorables, ont creusé de nouvelles inégalités de fortune en créant des moins-values pour certains. Mais il n'existe pas d'étude sur ce thème pour la France, alors que les panels de revenu et de patrimoine aux États-Unis (9) montrent que les ruptures des ménages (divorce, veuvage, etc.) constituent de loin les principales sources de variation du patrimoine au cours du temps.

Les données de l'ISF permettent de compléter ce tableau des inégalités de richesses fin 2003 (Cordier et al., 2006). À cette date, 300000 foyers fiscaux dont le patrimoine excédaient 720000 euros étaient redevables de l'impôt sur la fortune, soit environ 1,2\% des ménages. Les $10 \%$ les plus riches de ces assujettis (soit les $0,1 \%$ des ménages les plus riches) déclaraient le tiers de la masse totale imposable et les $1 \%$ les plus fortunés $12 \%$. Il y a donc autant d'inégalités au sein des plus riches qu'au sein de toute la population.

\section{La composition et la structure des patrimoines}

Les prédictions de la théorie des choix de portefeuille «standard» (Merton, 1971) concernant la diversification (portefeuille complet) et la composition des patrimoines (tous les épargnants détiennent, même en faible quantité, des actifs risqués et ceci quel que soient l'âge et la richesse des individus) sont loin de décrire la réalité.

En effet, toutes les enquêtes Patrimoine montrent que très peu de ménages possèdent la totalité des actifs recensés, que ce soit au niveau du patrimoine global ou à celui du seul portefeuille financier. De plus, à niveau de diversification donné, il y a rarement un portefeuille type. Enfin, la demande (instantanée) d'actifs risqués, notamment, est plutôt concave avec un maximum vers 40 ans (Guiso et al., 2003).

Mis à part les livrets d'épargne diffusés très largement au sein de la population (plus de huit ménages sur dix en possédaient au moins un, avant même que le système bancaire dans son ensemble ait reçu l'autorisation de distribuer ce type de produits), c'est le logement principal qui figure le plus fréquemment dans le patrimoine des ménages, en 2004 tout comme en 1998: $53 \%$ en étaient détenteurs, $56 \%$ en comptant les accédants à la propriété (Cordier et Rougerie, 2004). En 2004, l'épargne de long terme (assurances-vie et épargne retraite volontaire) concernait près de $44 \%$ des foyers (Darmon et Pagenelle, 2005) et l'épargne logement (compte ou plan) $41 \%$. Les actifs risqués étaient par contre nettement moins diffusés, toutefois un peu plus qu'en 1998 (13\% détenaient alors des titres directement et 10,5\% des OPCVM) : seuls $16 \%$ des ménages possédait des actions directement et environ 10,5\% des Sicav ou FCP. Enfin, l'épargne salariale concerne de plus en plus de ménages : $17 \%$ en détenaient contre $13 \%$ en 1998.

Là encore, une comparaison internationale est riche d'enseignements. Au début des années 2000, on dénombrait $15 \%$ d'actionnaires (directement ou indirectement) en Italie, $25 \%$ aux Pays-Bas, $34 \%$ au Royaume-Uni et $48 \%$ aux États-Unis (Guiso et al., 2003).

Selon les déclarations des ménages vivant en France, le patrimoine en montant se répartit entre $20 \%$ d'actifs financiers, $66 \%$ de biens immobiliers et $14 \%$ de biens professionnels. Cette structure varie fortement avec le niveau de la richesse : chez les plus riches, la part de l'immobilier diminue au profit des actifs financiers, notamment des valeurs mobilières.

Ces chiffres sont sensibles aux traitements statistiques destinés à corriger les déclarations des biais qui les affectent. Comme on l'a déjà souligné, l'ampleur de ces biais est importante. Les calages

9. Le Panel Study on Income Dynamics (PSID) du Michigan, par exemple. 
traditionnellement effectués, en prenant à quelques détails près la comptabilité nationale comme référence, tiennent compte de taux de sous-estimation différents selon les produits mais reposent sur l'hypothèse hardie d'une sous-estimation homogène selon les ménages. Sous ces hypothèses, le redressement conduirait à une structure du patrimoine assez différente : $37 \%$ du patrimoine serait investi en actifs financiers (au lieu de $20 \%$ ), $48 \%$ en immobilier et $15 \%$ en biens professionnels. Mais ces chiffres sont eux-mêmes à prendre comme des ordres de grandeur car ils seraient susceptibles de varier si on était en mesure d'affiner la méthode de calage (10).

\section{L'endettement}

L'accès au marché du crédit est un élément important de la stratégie inter-temporelle de consommation du ménage (rationnel) puisque c'est par l'épargne et l'endettement qu'il pourra adapter son plan de dépenses optimal en déconnectant le profil de ses consommations de celui de ses revenus. Cette dimension est essentielle pour l'acquisition de biens durables comme le logement.

En France, en 2004, près d'un ménage sur deux était endetté à un titre ou à un autre, dont un sur cinq pour l'acquisition de sa résidence principale et trois sur dix pour des achats de consommation. Environ un ménage sur dix cumulait endettement immobilier et crédit à la consommation. En volume, l'endettement pour le logement représentait $70 \%$ de la dette domestique des ménages (Houdré, 2007).

La proportion de ménages endettés a augmenté ces dernières années, passant de $43 \%$ en 1992 à près de $47 \%$ douze années plus tard. L'augmentation de l'encours d'endettement des particuliers, observée à partir des données de la comptabilité nationale, s'explique donc en partie par une diffusion accrue du crédit. Les ménages endettés sont plutôt issus des catégories aisées et sont en majorité des ménages jeunes, accédants à la propriété et disposant de peu de liquidités.

En 2004, la dette moyenne par ménage endetté atteignait 40000 euros, ce qui conduisait à des charges de remboursement annuelles d'environ $21 \%$ des revenus disponibles (Houdré, 2007). Mais pour $7 \%$ des ménages (1,8 million) cette charge dépassait $30 \%$, ce qui les exposait au risque de surendettement (11).

\section{Les transmissions intergénérationnelles entre vifs ou au décès}

En matière de transferts intergénérationnels, la théorie économique distingue deux grands types de motivations : les legs accidentels, transferts involontaires dus à l'incertitude sur la durée de vie et à l'imperfection du marché des rentes viagères, qui ne donnent lieu qu'à des héritages ; et les transferts volontaires, qui répondent à un véritable motif de transmission familiale, et qui se traduisent à la fois par des transferts entre vifs et au décès (12).

Les données successorales sont adéquates pour fournir un panorama des héritages, les enquêtes auprès des ménages étant plus précises et plus larges en ce qui concernent les aides ou donations entre vivants.

10. On retrouve les mêmes écarts au sein du seul patrimoine financier : les données brutes d'enquête conduisent à $18 \%$ de livrets (15\% en redressé selon la comptabilité nationale), $15 \%$ en épargne logement (resp. $12 \%$ ), $20 \%$ en valeurs mobilières (resp. $17 \%$ ) et $27 \%$ en assurances-vie (resp. $36 \%$ ). Ce sont donc les produits d'assurance et de préparation de la retraite qui représentent la part la plus importante des avoirs financiers des ménages (Girardot et Marionnet, 2007). Là encore, cette structure varie avec la richesse des ménages, la part des actions étant beaucoup plus importante chez les plus fortunés.

11. Rappelons qu'en 2003 plus de 165000 dossiers avaient été déposés dans les commissions de surendettement (en 2008, leur nombre était monté à près de 183000 ).

12. Voir Arrondel et Masson (2006). Les motivations peuvent être de différente nature : logique altruiste si les parents veulent aider leurs enfants dans les besoins ou rapprocher les niveaux de vie entre les générations; logique d'échange si les parents rémunèrent leurs héritiers de leurs services ; logique paternaliste pour la seule satisfaction de laisser quelque chose à ses enfants. 
Dans les années 1980, selon les données de la Direction Générale des Impôts, seul un décès sur deux conduisait à une déclaration de succession. En 1994, 60 \% des décès avaient donné lieu à une déclaration. Pour l'année 2000, l'administration fiscale avait enregistré les successions de $2 / 3$ des personnes décédées pendant l'année : au total, un cinquième seulement des successions entre parents et enfants était redevable de droits. Au cours des 20 dernières années, l'âge moyen au décès a augmenté de cinq ans et celui des héritiers a suivi la même évolution : en 2000, l'âge moyen des personnes héritant d'un ascendant (premier ou second décès) était de 47 ans. Néanmoins, les familles semblent avoir fait des efforts pour compenser ce retard en anticipant les transferts volontaires : l'âge moyen des donateurs a moins augmenté au cours de la période et (différence d'âge intergénérationnelle aidant) l'âge moyen des donataires a même diminué, passant de 39 ans à 37,5 ans.

En moyenne en 2000, tous héritiers confondus, les défunts français dont la succession était déclarée (13) laissaient 99000 euros à leurs légataires (95000 euros pour les seuls enfants héritiers). En 2006, cette somme s'élève à 175000 euros; cette augmentation étant principalement due à l'évolution des prix de l'immobilier sur la période. Mais, comme pour le patrimoine, les disparités étaient très importantes : plus de 500000 euros sont transmis en moyenne chez les $10 \%$ les plus riches et près de 1800000 euros en moyenne dans le centile supérieur (Arrondel et Masson, 2007a).

Selon l'enquête Patrimoine 2004, parmi les ménages ayant des enfants vivant hors du domicile parental, un sur huit déclarait avoir déjà fait une donation entre vifs (Cordier et al., 2007). Cette pratique est plus fréquente parmi les agriculteurs (plus de $40 \%$ ) et les travailleurs indépendants (environ $15 \%$ ) qui l'utilisent pour transmettre leur outil de travail. Les montants donnés étaient donc relativement importants : une donation sur dix s'élevait à plus de 100000 euros (deux sur dix chez les chefs d'entreprise et les professions libérales). Par ailleurs, environ un Français sur deux avait aidé ou aidait ses enfants sous la forme de don, de versements ou de prêt d'argent ou encore de mise à disposition d'un logement. Dans six cas sur dix, cette aide a été fournie pendant les études des enfants.

\section{Pourquoi les ménages épargnent en France : des éclairages nouveaux}

$\mathbf{P}$ ar rapport aux autres pays occidentaux, la France se distingue par un fort déséquilibre intergénérationnel, en termes d'écart de niveau de vie et plus particulièrement de patrimoine : les seniors et les plus âgés ont bénéficié d'une hausse de leurs revenus et de leurs avoirs alors que les ménages les plus jeunes ont vu leurs conditions de vie relatives se détériorer ou leur projet d'accumulation (pour le logement) se dérouler avec retard. En France, l'épargne, de niveau élevé par rapport aux autres pays, est en outre inégalement répartie, étant très concentrée au sein des classes d'âge de 50 à 70 ans - et même au-delà - avec cependant de fortes disparités au sein même de ces âges. Certains semblent avoir accumulé trop peu, si bien que leur consommation accusera une baisse sensible au moment du passage à la retraite. D'autres épargneraient « trop », possédant à un âge élevé des patrimoines bien supérieurs à ce que l'on observe dans d'autres pays développés - en dépit d'un système de protection sociale jugé plutôt généreux à l'échelle internationale - et souvent trop élevés pour leur propre consommation sur leurs vieux jours.

Lors d'une phase de croissance soutenue, cette concentration des biens aux mains des aînés n'est peut-être pas trop gênante. Mais en période de crise, elle constitue un handicap : il serait probablement préférable pour l'économie qu'une part plus importante du patrimoine soit détenue par des personnes plus jeunes ou d'âge moyen.

13. Au premier décès au sein d'un couple, la somme léguée correspond le plus souvent à environ la moitié du patrimoine du ménage. 
Une question clef concerne alors l'explication de cette sur-épargne d'une fraction importante des seniors en France. Plusieurs explications peuvent être avancées. Les circonstances historiques et les effets de génération propres à notre pays en constituent une partie. Une autre part peut être due aux spécificités des motifs d'épargne et des comportements d'accumulation de nos compatriotes : il s'agirait de savoir si l'accumulation élevée des seniors français provient principalement de l'épargne constituée pour les vieux jours, de l'épargne de précaution face aux aléas de la vie, des caractéristiques spécifiques du processus d'accession à la propriété du logement - apport personnel, remboursements d'emprunt (14) - ou encore du désir de transmettre des biens à ses enfants ou petits-enfants.

Au-delà des comparaisons internationales, qui demeurent fondées sur des enquêtes d'opinion fragiles dont les résultats sont parfois difficiles à interpréter, les données récemment collectées (qu'elles proviennent d'enquêtes ou de sources fiscales), et leurs nombreuses exploitations dont on vient de rendre compte, ont déjà permis de répondre à une partie de ces interrogations, mais une partie seulement. Les études présentées dans ce numéro constituent à cet égard un complément précieux : bien qu'elles reposent souvent sur des approches historiques ou sur des sujets relativement pointus, elles abordent néanmoins à plusieurs reprises des questions en phase avec les interrogations que se posent actuellement les milieux académiques, politiques ou professionnels concernés.

Est-on plus prudent ou plus averse au risque en France qu'à l'étranger? Est-on particulièrement marqué par le désir de propriété immobilière, résurgence de l'attachement à la terre dans un pays de tradition paysanne ? Le motif de transmission est-il plus prégnant qu'ailleurs dans notre pays de culture catholique et latine?

Même s'il n'apporte pas de réponse définitive à toutes ces interrogations, le dossier présenté renseigne, de façon tout à fait opportune, sur chacune des quatre motivations d'épargne évoquées (retraite, précaution, immobilier, transmission) dans le paysage français d'hier et d'aujourd'hui.

\section{Une épargne pour les vieux jours inégalement répartie}

La difficulté d'identifier les motifs de détention des ménages tient à une double constatation. D'une part, un même actif peut satisfaire différents besoins : l'assurance-vie (en cas de vie), peut par exemple aussi bien servir d'épargne de précaution à long terme, pour la retraite, mais aussi à la transmission grâce à la contre garantie décès. D'autre part, les motifs de détention ou leur hiérarchie varient sensiblement d'un ménage à l'autre. Si l'on excepte, et encore, les produits dédiés à telle ou telle fonction, la composition du patrimoine renseigne donc peu sur les mobiles qui ont prévalu à son accumulation.

La voie la plus directe pour connaître les motivations d'épargne des ménages consiste alors à les interroger explicitement sur les motifs de détention de chaque actif qu'ils possèdent. C'est ce que permet l'enquête Patrimoine 2004. À partir de ces informations, Amandine Brun-Schammé et Michel Duée tentent ainsi d'isoler l'épargne en vue de la retraite au sein du patrimoine financier. Ils définissent tout d'abord une épargne financière (dite) de long terme, qui écarte les liquidités et quasi liquidités (concernant en priorité le court et moyen terme), les produits liés à l'accession à la propriété (épargne-logement) et les assurances décès (pour la transmission). Cette épargne financière de long terme regroupe en première approximation les Pep (à l'époque), les assurances-vie (en cas de vie), les valeurs mobilières et les produits dédiés, d'épargne retraite proprement dit. Au sein de cette composante du patrimoine, l'épargne en vue de la retraite regroupe

14. On sait que le logement occupe une place à part dans les patrimoines et les projets patrimoniaux des Français : le désir d'être propriétaire constitue un idéal prioritaire chez la plupart de nos compatriotes. Mais c'est aussi le cas d'autres pays comme l'Espagne. 
les actifs pour lesquels le ménage a indiqué un tel motif de détention (éventuellement parmi d'autres) (15).

Les produits retraite sont, sans surprise, les actifs les plus détenus en prévision de la retraite, mais toutefois dans moins de $80 \%$ des cas. À l'inverse le motif retraite ne dépasse pas $20 \%$, en moyenne, dans le cas de la détention des valeurs mobilières. Mais, pour chaque actif, il est maximal aux âges mûrs (de 45 à 60 ans). Qui épargne alors le plus pour la retraite ? Là encore les ménages d'âge mûr, mais aussi les indépendants, moins bien couverts que les salariés ; le revenu n'a, lui, pas d'effet significatif. En revanche, la détention d'épargne financière de long terme, peu sensible à l'âge, augmente fortement avec le revenu.

Une autre manière de juger de l'importance de l'épargne en vue de la retraite consiste à analyser la réponse des ménages à l'offre de produits spécifiquement destinés à cet usage. Alexis Direr étudie le cas récent du Perp, produit qui contraint à une sortie en rente viagère au moment du passage à la retraite. La rente viagère est traditionnellement peu diffusée, sa désaffection s'expliquant par de multiples facteurs tels « l'antisélection » (les détenteurs espèrent vivre plus longtemps que les autres), ou encore la crainte que ce type de placement puisse être interprété comme un refus délibéré de léguer à ses enfants. Dans ce contexte, le succès du Perp, bien que limité, semble réel puisqu'il s'est diffusé dans toutes les classes sociales et que le nombre de plans ouverts fin 2007 s'élevait déjà à 2 millions. En outre, si les encours actuels restent faibles, ils sont appelés à grossir régulièrement puisque le Perp « lie de façon irréversible les cotisants jusqu'à leur décès » - sachant que l'âge moyen de ces derniers se situe autour de 40 ans.

Les implications techniques d'un produit complexe (cantonnement, taux d'actualisation nul pour le premier versement, fiscalité, etc.) n'ont cependant pas toujours été bien comprises. Tout d'abord, la volonté des assureurs d'éviter une baisse nominale des rentes versées au cours de la retraite fait que le Perp est plutôt une épargne des très vieux jours : le montant de la rente pourrait doubler entre 60 et 83 ans... Les différences d'espérance de vie, entre classes sociales ou entre sexes, jouent donc un rôle considérable. Avant janvier 2007, il y avait ainsi une seule table de mortalité, plutôt avantageuse pour les cotisants. Depuis, il y en a deux, distinguant hommes et femmes, et les espérances de vie ont été revues à la hausse : l'opération s'est révélée presque neutre pour les hommes mais a fait perdre 8 à $15 \%$ de rentes aux femmes, sans que d'ailleurs cette perte n'ait eu d'effet apparent sur leur participation. Par ailleurs, la mortalité sociale différentielle se traduit, chez les hommes, par des écarts de taux de rendement interne considérables entre les cadres et les ouvriers. Enfin, la fiscalité de ce type de produit - déductions des cotisations, taxation des rentes - génère des effets complexes, régressifs aussi bien que progressifs, d'une ampleur potentiellement comparable à ceux engendrés par les écarts d'espérance de vie.

Le Perp, par les engagements irréversibles qu'il comporte, préfigure en quelque sorte l'épargne en vue de la retraite de demain. Mais qu'en était-il de cette épargne hier ? Jérôme Bourdieu et Lionel Kestenbaum étudient la question de l'épargne adéquate pour les vieux jours (adequacy of saving) à une époque, entre 1820 et 1940, où n'existaient pas, ou encore peu, de pensions publiques. Ils distinguent les personnes qui avaient accumulé suffisamment pour satisfaire une consommation minimale pendant leurs vieux jours selon trois critères alternatifs :

- un niveau absolu de 4000 francs ;

- une fraction donnée du PIB par tête au moment de l'entrée en retraite, non indexée par la suite ;

15. Ces décompositions sont certes relativement frustes et les déclarations de motivation en questionnaire fermé sont parfois sujettes à caution, mais on ne peut espérer faire mieux tant que l'on ne procède pas à des entretiens libres en profondeur, particulièrement longs et coûteux. 
- une fraction donnée du PIB par tête tout au long de la retraite, soit une consommation indexée sur la croissance économique (16).

Les auteurs, qui s'appuient sur les données uniques de l'enquête TRA (17), parviennent à deux conclusions générales : non seulement les ouvriers, mais aussi une bonne part des classes moyennes, n'avaient pas les moyens nécessaires ; et la situation des plus âgés (mesurée par la part de ceux qui ont des moyens suffisants) « s'améliore continûment au cours du XIX $X^{e}$ avant de connâेtre une dégradation brutale dans les années $1900 »-$ avec de fortes inégalités entre hommes et femmes ou entre milieux sociaux.

Comment interpréter le second résultat, apparemment surprenant, et d'autant plus accusé si l'on veut maintenir une parité entre actifs et retraités ( $3^{\mathrm{e}}$ critère) ? L'allongement de l'espérance de vie, plus marqué et précoce pour les femmes, n'a que des effets lents et limités. L'explication principale réside dans l'effet d'hystérésis du patrimoine, variable de stock, confronté aux cycles longs et aux crises économiques : le pire est d'avoir produit son effort d'accumulation pendant des années de stagnation (comme à la fin du XIX ${ }^{\mathrm{e}}$ ) et d'être âgé au moment d'un boom économique (comme au début du XXe à la Belle Époque). Une forte dévalorisation des actifs à la veille de la retraite (guerre de 1914-1918) produit également des effets dévastateurs. Comment contrer de tels effets pervers? Par une mutualisation intergénérationnelle des risques que permet normalement la retraite par répartition.

\section{L'attitude face au risque : épargne de précaution et gestion du portefeuille}

Les attitudes des ménages à l'égard du risque influencent leurs comportements patrimoniaux à travers trois canaux principaux :

- la gestion du portefeuille et le choix d'actifs selon le risque et le rendement et d'autres critères (la fiscalité différentielle, la liquidité, les coûts d'information, de gestion ou de transaction, etc.) ;

- l'épargne de précaution, moyen d'auto-assurance contre les divers risques de l'existence, concernant le revenu, la longévité, la santé et la dépendance ;

- la demande d'assurance contre ces mêmes risques, qui dépend notamment de la couverture publique.

La caractéristique principale des portefeuilles français est d'être à la fois très divers et peu diversifiés. Si les petits patrimoines apparaissent essentiellement composés de liquidités et quasiliquidités, les grosses fortunes, à l'inverse, sont largement diversifiées. Il existe cependant, aujourd'hui comme hier, un grand nombre de combinaisons d'actifs différentes au sein des niveaux de richesse intermédiaires, adoptées chacune par une fraction significative de la population (Arrondel et Masson, 2007b). Si la composition du patrimoine est principalement liée à la taille du patrimoine global, ce qui s'explique par des facteurs bien connus (effets de seuil, indivisibilités, coûts de détention, etc.), le faible niveau de diversification résulte également de la diffusion limitée de certains placements (comme les valeurs mobilières ou les rentes viagères), qui constituent autant d'énigmes pour la théorie économique et la rationalité standard.

La détention limitée des actions est le problème qui a le plus préoccupé les économistes. En France, comme on vient de le voir, elle ne concerne que 15 à $20 \%$ des ménages, et la détention directe ou « indirecte » d'actions, i.e. par l'intermédiaire de fonds communs de placement, Sicav,

16. Le $3^{e}$ cas le plus avantageux, correspondrait à l'avant 1993 (réforme Balladur), le $2^{e}$ à la situation actuelle.

17. L'échantillon de l'enquête 3000 familles (ou enquête TRA) est constitué par les individus dont le patronyme commence par les lettres $T, R$ et $A$, décédés après 1820, pour lesquels on dispose d'un ensemble de renseignements sur la situation au décès. 
PEA..., ne dépasse guère le quart de la population ; en outre, la part du portefeuille consacrée à ces actifs demeure souvent limitée. Une demande aussi modeste s'explique mal alors que l'on observait un différentiel positif important durable entre les taux de rendement des actions et celui des bons ou obligations. Ce paradoxe dit de «l'énigme de la prime de risque » a toujours été plus important aux États-Unis que dans notre pays (18). Il peut paraître déplacé de l'évoquer dans la période de crise financière que nous connaissons : la situation actuelle nécessiterait de fait un renouvellement des études, consacrées à l'existence et à l'ampleur de ce phénomène.

L'autre enjeu majeur aujourd'hui concerne le volume de l'épargne de précaution face aux différents risques de l'existence, qu'il s'agisse du risque de chômage, de la viabilité du système de retraite, du poids croissant des dépenses de santé et de dépendance, ou encore des inquiétudes suscitées par l'avenir professionnel des enfants.

Mesurer cette épargne de précaution s'avère une entreprise délicate qui supposerait de connaître ce que serait le comportement des ménages dans un monde où le système de retraite serait parfaitement viable, la dépendance maîtrisée, etc. Luc Arrondel et Hector Calvo-Pardo envisagent le cas le plus balisé, celui du risque sur le revenu, sous deux formes : rémunérations aléatoires ou risque de perte d'emploi. La question que les auteurs se posent est la suivante : quelle part de la richesse des ménages peut être attribuée au «patrimoine de précaution », i.e. au patrimoine qui disparaîtrait si le risque pesant sur le revenu pouvait être supprimé.

La réponse suppose déjà de contrôler par une mesure individuelle de l'aversion pour le risque (ou de la " prudence ») - disponible dans l'enquête Patrimoine 2004 : les indépendants, par exemple, ont des revenus plus aléatoires que les salariés mais pas forcément une épargne de précaution supérieure du fait d'une moindre aversion au risque. La littérature consacrée montre par ailleurs que les estimations empiriques dépendent de l'indicateur d'exposition au risque considéré : elles sont beaucoup plus élevées si l'on choisit d'estimer le risque « revenu » à partir des observations passées mesurées en panel que si l'on se fonde sur les anticipations subjectives (plus pertinentes) déclarées par les ménages eux-mêmes. Luc Arrondel et Hector Calvo-Pardo utilisent les anticipations à cinq ans de risque de chômage ou d'évolutions possibles du revenu que fournit l'enquête Patrimoine 2004. Les résultats obtenus, variables selon les indicateurs ou la méthode d'estimation, s'étagent en général entre $1 \%$ et $10 \%$ du patrimoine accumulé, valeurs assez faibles mais en accord avec les estimations étrangères utilisant les anticipations de revenu.

Ces estimations ne sont toutefois que des minorants : elles ne concernent que le risque « revenu ", et non les inquiétudes à l'égard de l'avenir des retraites, de la santé et de la dépendance. Par ailleurs, des valeurs trop élevées ne seraient guère plausibles : une épargne de précaution représentant $80 \%$ du patrimoine moyen, par exemple, signifierait que les riches s'avèreraient les plus à plaindre, les plus peureux ou les plus mal couverts contre les risques de l'existence...

\section{Accéder à la propriété de son logement : une décision du couple}

Cédric Houdré s'intéresse à ce qui constitue l'épine dorsale des comportements patrimoniaux pour la plupart des ménages, à savoir l'acquisition du logement principal. La demande de cet actif spécifique résulte d'une interaction complexe entre de nombreux déterminants :

18. Les études françaises existantes aboutissent à des résultats empiriques contradictoires. Pour Tanay (2002), un investisseur qui, à la veille de la guerre de 1914, aurait placé 1000 francs en actions aurait vu son capital multiplié par 31 (en valeur réelle) à la veille de l'an 2000, alors qu'il n'aurait récupéré que 400 francs de sa mise initiale s'il avait acheté des obligations. Pour Le Bris et Hautcoeur (2008), qui utilisent d'autres pondérations pour le calcul des indices boursiers, l'évolution des cours boursiers français de 1854 à 2007 montrerait que les actions auraient enregistré un rendement réel positif au XIX', mais négatif au XX' siècle (en raison de l'inflation et des deux guerres mondiales) : sur cette période, l'écart limité avec le rendement des obligations serait alors compatible avec une prime de risque standard. 
- la quasi-indivisibilité de l'investissement fait jouer à l'endettement un rôle central ;

- les décisions concernent un bien collectif du couple qui l'engage sur le long terme alors que la stabilité des unions n'est pas forcément assurée à cet horizon ;

- le logement principal revêt de façon indissociable la nature d'un bien de consommation, d'une prestation de services d'habitation et d'un bien d'investissement, réserve de valeur ;

- les avantages fiscaux, directs ou indirects, liés à la propriété interviennent également dans le processus de décision entre location et achat du logement, etc.

Enfin, sur une échelle temporelle aussi longue - celle de la constitution progressive de l'apport personnel jusqu'à la fin des remboursements d'emprunt - l'offre de travail des femmes (au sein d'un ménage accédant à la propriété) ne peut être considérée comme exogène : elle résulte d'une décision, parallèle au choix du taux d'endettement.

Gardant à l'esprit cet enchevêtrement de multiples facteurs, l'auteur s'interroge sur le devenir de l'accession à la propriété face aux évolutions possibles des marchés du crédit et de l'emploi en s'attachant aux liens, a priori réciproques, entre contraintes d'endettement et flexibilité de l'offre de travail. Il considère plus particulièrement la relation, au sein des couples de l'enquête Patrimoine 2004, entre le taux d'effort (pourcentage du revenu du ménage consacré aux remboursements d'emprunt) et la composante qu'il estime la plus flexible de l'offre de travail, à savoir le taux d'activité féminine.

Sachant que de nombreux comportements influent simultanément sur la décision d'activité de la femme, comment contourner les inévitables problèmes d'endogénéité et de causalité réciproque ? L'auteur se tire de cette difficulté en utilisant une relation causale non linéaire entre le taux d'effort et l'activité féminine (qui augmente seulement au-delà d'un certain seuil de taux d'effort) : les contraintes sur le marché du crédit entraineraient donc bien une participation accrue des épouses au marché du travail. L'article quantifie alors l'impact sur l'accession que pourrait avoir un marché du travail dégradé en présence d'un marché du crédit restreint.

\section{La transmission du patrimoine, vecteur de reproduction des inégalités entre générations ?}

Déjà soulignée dans l'introduction au numéro spécial d'Économie statistique de 1996, cette question est certainement celle qui intéresse le plus les sociologues : de nombreux travaux ont exploré les différentes dimensions de l'héritage (entendu au sens large incluant toutes les formes de transmission et pas seulement l'héritage matériel à l'occasion d'un décès), que ce soit sous l'angle de l'équité des legs entre héritiers ou en se focalisant sur les mécanismes par lesquels ces transmissions sont susceptibles d'influer sur le devenir du récipiendaire. Le présent dossier apporte deux nouvelles pierres à l'édifice.

À partir des données historiques (1820-1940) de l'enquête $T R A$, évoquée plus haut, Jérôme Bourdieu, Gilles Postel-Vinay et Akiko Suwa-Eisenmann s'intéressent à la reproduction des inégalités de fortune d'une génération à la suivante en comparant, à différentes périodes, la fortune laissée par le père à celle laissée par le fils - lorsque les deux sont positives. La mesure utilisée est soit la corrélation intergénérationnelle entre les deux montants (IGC), soit l'élasticité intergénérationnelle (IGE), les deux indicateurs étant égaux en cas de régime permanent (c'est-à-dire en cas d'inégalité stable entre deux générations). Le modèle de référence en la matière est celui, déjà ancien, de Becker et Tomes (1986) qui se placent dans une telle situation d'équilibre. Ce modèle suppose des parents altruistes qui cherchent à lisser la consommation et les ressources entre les différentes générations de la famille. Les 
parents les plus aisés ont en moyenne des enfants moins bien lotis qu'eux-mêmes du fait de la régression vers la moyenne des revenus humains : le legs sera alors utilisé comme un rempart ou un «tampon » (buffer) compensatoire en vue d'assurer aux enfants un niveau de vie comparable au leur, si bien que le degré d'immobilité intergénérationnelle des fortunes est élevé parmi les plus riches (19). En revanche, les parents moins favorisés ayant en moyenne des enfants mieux dotés qu'eux-mêmes, afin de parvenir au lissage intergénérationnel de la consommation, il faut qu'ils laissent à leurs descendants des " héritages négatifs ", à savoir des dettes, un passif, etc. Il s'ensuit que la mobilité intergénérationnelle en termes de consommation ou de fortune est beaucoup plus forte en bas et au milieu de l'échelle sociale ou de celle du patrimoine (20).

Les trois auteurs divisent la période 1820-1940, qui a connu des bouleversements historiques, politiques, technologiques considérables en quatre sous-périodes plus homogènes. Au premier regard, les résultats semblent pourtant indiquer que l'on n'est pas très éloigné d'une situation de régime permanent. Sur l'ensemble de la population, les deux indicateurs d'immobilité des fortunes (IGC et IGE) ont en effet des valeurs proches, qui fluctuent en outre assez peu d'une sous-période à l'autre, autour de 0,4. Par ailleurs, les prédictions du modèle de Becker et Tomes paraissent corroborées : sur l'ensemble de la période, l'élasticité intergénérationnelle (IGE) est de 0,81 pour le quartile supérieur des richesses, mais seulement de 0,16 au sein des trois autres quartiles. Un examen plus poussé montre cependant que, après 1914, cette même élasticité IGE a nettement baissé chez les plus riches « sous l'action conjointe des guerres, de l'inflation et de la fiscalité » mais plutôt augmenté dans le reste de la population. Les auteurs se proposent de tester la robustesse de ces évolutions en introduisant encore d'un côté, les riches héritiers qui ne laissent rien, et de l'autre, les transmetteurs qui n'ont rien reçu (21).

Ces mesures de l'immobilité intergénérationnelle des fortunes renvoient à une question plus générale : comment les différentes formes d'héritage humaines et non humaines, et plus particulièrement les transmissions patrimoniales, influencent-elles le statut et les comportements économiques des héritiers? Du fait de la richesse de leurs données rétrospectives et biographiques, les enquêtes patrimoniales de l'Insee, tout particulièrement celle de 2004, nous permettent d'apporter quelques éléments de réponse à ce type de question.

Sibylle Gollac s'attaque dans ce cadre à un sujet difficile, celui de la transmission du statut d'indépendant. L'analyse est rendue complexe du fait qu'elle relève de registres très divers. La première difficulté vient sans doute de ce que l'on cherche à expliquer un état qui peut être l'aboutissement d'un parcours comprenant plusieurs phases et que l'on n'observe pas toutes les personnes au même moment de ce parcours (on peut commencer par être salarié dans l'entreprise familiale avant d'être appelé à en devenir le chef) : l'effet de l'âge, surtout avant 40 ans, est donc difficilement interprétable. Un second point épineux, comme à chaque fois que l'on s'intéresse à un comportement structurant le cycle de vie de la personne, concerne l'hypothèse selon laquelle certaines caractéristiques de l'individu s'imposent à lui comme une contrainte exogène : ainsi le diplôme est-il certainement le résultat d'un investissement choisi de façon cohérente selon ses ambitions professionnelles ou les projets que ses parents peuvent nourrir à son endroit. Enfin, une partie des thèses sociologiques que voudrait tester l'auteur nécessiterait des données plus précises sur la génération antérieure que celles dont on dispose au travers de l'enquête Patrimoine.

19. "Assets bequeathed to children in richer families act as a buffer to offset any intergenerational regression to the mean in the earnings of children [...] As a result, bequest could regress away from the mean » (p. S21).

20. Le lecteur intéressé pourra se reporter à Masson et Pestieau (1991), notamment le supplément 3

21. C'est ce que font Arrondel et Grange (2006) à partir des mêmes données historiques mais portant uniquement sur la Loire-Inférieure (actuelle Loire-Atlantique). En considérant toute la population du département(riches ou non, héritiers ou non), ils obtiennent une élasticité (IGE) de l'ordre de 0,45. 
Sybille Gollac a tout à fait conscience de ces limites puisqu'elle complète son analyse statistique par une partie plus qualitative - un cas d'école - qui fait bien ressortir la complexité du phénomène. Elle met notamment en évidence que les facteurs explicatifs de la transmission du statut d'indépendant ne se limitent pas, tant s'en faut, aux caractéristiques du patrimoine professionnel, mais dépendent encore de l'existence d'autres biens patrimoniaux et, plus généralement, de l'ensemble des efforts consentis par les parents. La reconnaissance du rôle du capital humain informel, en plus de celui joué par les dimensions financières, est sans doute une des conclusions les plus importantes de l'étude. L'auteur souligne par ailleurs que les inégalités de transmission au sein de la fratrie sont en l'état difficiles à appréhender. L'héritier de l'entreprise est-il avantagé ou pénalisé ? La réponse variera sensiblement selon le contexte familial spécifique et le secteur d'activité considéré, agricole, artisanal ou libéral.

Dans une autre étude utilisant les mêmes données rétrospectives et biographiques de l'enquête Patrimoine 2004, Arrondel et Masson (2009) ont tenté de mesurer l'effet des donations, ou plus généralement de transmissions précoces sur les choix d'investissement des enfants bénéficiaires - acquisition du logement, création ou reprise d'une entreprise. Les économistes distinguent au plan théorique deux cas polaires. Le premier, dit « effet Carnegie », est celui où les dons (ou héritages) déjà reçus entraînent une augmentation de la seule consommation courante - mais pas des projets à plus long terme - et engendrent par ailleurs une baisse de l'offre de travail. Le cas opposé est celui où un transfert patrimonial précoce favorise au contraire l'insertion du jeune bénéficiaire dans différents domaines : accession à la propriété, mise en ménage ou création d'une famille, obtention d'un métier stable et même, pour certains, réalisation d'un projet d'entreprise ; l'idée est que le transfert précoce permettrait soit de lever les contraintes de crédit, soit de financer en partie le projet en question.

Les résultats penchent clairement en faveur du second scénario. Prenons le cas du statut d'entrepreneur, qu'il s'agisse d'une création d'entreprise ou d'une reprise, hors le cas d'une entreprise familiale (pour écarter précisément les spécificités de la transmission professionnelle étudiée par Sibylle Gollac). Au sein de la population non agricole, on dénombrait 4,3\% de créateurs d'entreprise et $2,8 \%$ de repreneurs. Parmi les différents transferts intergénérationnels (dons ponctuels d'argent, versements réguliers d'argent, donations, héritages), c'est seulement le fait d'être donataire qui favorise le passage au statut d'entrepreneur. La probabilité de créer une entreprise est de $4,1 \%$ sans l'aide d'une donation, elle s'élève à $6,5 \%$ pour les donataires ; si l'on cumule les créations et les reprises, les pourcentages passent respectivement de $6,9 \%$ à $9,3 \%$. En revanche, les espérances d'héritage ou de donation n'ont pas d'effet. Parmi les autres déterminants de la création d'entreprise, on constate qu'être fils ou fille d'entrepreneur favorise très fortement le désir de l'être soi-même : la probabilité de devenir entrepreneur est quatre fois supérieure à celle des enfants de salariés. Un diplôme élevé y prédispose également. On retrouve ici l'idée, développée par Sibylle Gollac, que la réussite d'un projet entrepreneurial est grandement facilitée par le cumul d'héritages matériel et humain.

Enfin, si les transmissions précoces apparaissent aussi utiles aux bénéficiaires, comment les favoriser sans renforcer la reproduction des inégalités d'une génération à la suivante (les donations sont une pratique de personnes fortunées) ? On sait que les seniors français réagissent aux avantages fiscaux accordés aux seuls transferts entre vifs en augmentant de manière sensible les donations à leurs (petits-) enfants (Arrondel et Masson, 2009). C'est d'ailleurs pour cette raison, parmi d'autres, que de plus en plus d'avantages fiscaux à la donation sont accordés dans notre pays. Mais cette logique d'allégement fiscal semble avoir atteint ses limites. Si l'on entend miser sur la famille pour « fluidifier des retours patrimoniaux » vers les nouvelles générations, la solution appropriée consisterait, à contre-courant des évolutions actuelles, à augmenter l'impôt sur l'héritage : un alourdissement et une progressivité plus forte des droits de succession permettraient en effet d'accroître l'avantage fiscal différentiel accordé à la donation tout en évitant une trop forte reproduction des inégalités de fortune. 


\section{Perspectives}

Walgré sa richesse, qui a permis de conduire toutes les études précitées, l'information collectée sur le patrimoine n'est cependant pas encore tout à fait à la hauteur des attentes sociales. Le dispositif de mesure se devra donc d'évoluer dans les années à venir sous la double pression de la demande nationale (exprimée notamment dans les divers programmes à moyen terme du Cnis) et des recommandations internationales. La pression apparaît cependant moins forte que sur d'autres sujets : la thématique de l'épargne et du patrimoine n'est certes pas absente des demandes adressées au système statistique public, mais elle n'occupe qu'une place relativement limitée.

\section{Une demande sociale hésitante}

Il n'y a actuellement aucun règlement européen incitant les pays à une collecte harmonisée sur le sujet, alors qu'il en existe dans le domaine de l'emploi ou des revenus.

Ce n'est que tout récemment que la Banque Centrale Européenne a lancé le projet d'un tel ensemble d'opérations harmonisé au niveau européen. Mais à l'heure actuelle, les pays n'ont pas encore trouvé un terrain d'entente, en particulier au niveau de la taille de l'échantillon et de certains choix méthodologiques comme le fait de réaliser l'enquête en panel (i.e. avec plusieurs interrogations, sur plusieurs années pour le même ménage) ou sous la forme actuelle de « coupes instantanées » avec des échantillons différents pour chaque enquête.

Aucun des trois derniers programmes à moyen terme du Cnis ne privilégie par ailleurs l'amélioration des connaissances dans le domaine du patrimoine. De très nombreuses demandes sont liées à la connaissance des populations en difficulté : pauvreté, illettrisme, étrangers en situation irrégulière, personnes surendettées, jeunes en errance, toxicomanie. Le vieillissement est également très souvent cité, avec ses conséquences sur les transferts et la redistribution ainsi que les phénomènes liés à la discrimination (entre sexe, selon l'origine ethnique, etc.), la violence sous toutes ses formes (physique ou mentale, dans la rue, au travail ou au sein du foyer), les compétences ou encore la santé. Sur la période 2004-2008, quelques recommandations portaient sur une meilleure connaissance des revenus du patrimoine et de leur contribution à l'inégalité, ainsi que sur un éclairage particulier sur les extrêmes de la distribution des revenus (pauvreté et hauts revenus comme indicateurs imparfaits des plus grosses fortunes).

S'il est encore trop tôt pour connaître dans le détail le moyen terme 2009-2013, tout laisse à penser qu'il n'y aura pas d'innovation forte.

\section{Les nouveaux domaines statistiques à explorer}

Le défi qui s'offre actuellement au système statistique serait alors bien en continuité avec le passé récent : consolider les acquis récents malgré des difficultés grandissantes (baisse tendancielle de taux d'acceptation pour les enquêtes lourdes dans la plupart des pays occidentaux), améliorer la connaissance des montants en réduisant l'écart entre données d'enquêtes et estimations retenues dans la comptabilité nationale et surtout, et c'est là que réside le défi le plus difficile à relever, améliorer la connaissance du haut de la distribution.

La future enquête Patrimoine, programmée par l'Insee pour l'automne 2009, devrait ainsi bénéficier d'un échantillon complémentaire tiré parmi les ménages les plus riches et le questionnement sera enrichi d'un module destiné à mesurer certaines composantes immatérielles du patrimoine, comme le « capital social » dont les études de sociologues comme les Pinçon-Charlot (1996) ont montré l'importance dans cette strate de la population. 
Mais la collecte sera difficile : les ménages riches se distinguent en effet par des taux de réponse particulièrement bas, et ce dans tous les pays. Aux États-Unis, pays pourtant réputé pour l'aisance avec laquelle ses habitants abordent les questions pécuniaires, seulement environ $20 \%$ des ménages les plus fortunés répondent aux enquêtes.

L'importance du taux de non-réponse rend difficile la tâche du statisticien qui doit pondérer l'échantillon des répondants de façon à le rendre « représentatif » de l'ensemble de la population, ce qui est en l'occurrence une gageure, la diversité des comportements des personnes les plus riches rendant délicat le remplacement de l'une par l'autre. L'évaluation des actifs particulièrement présents chez les ménages les plus fortunés risque également d'être difficile (actions non cotées, patrimoine professionnel, œuvres d'art, résidences si exceptionnelles qu'elles n'ont pas d'équivalent sur le marché, etc.). La clef du succès résidera sans aucun doute dans l'utilisation conjointe de données fiscales, exhaustives mais portant sur une notion de patrimoine restreinte, et de données d'enquêtes qui ont l'avantage de cerner un patrimoine plus complet mais l'inconvénient d'être affectées par une non-réponse de grande ampleur.

\section{Le patrimoine des ménages à l'épreuve de la crise}

Mais l'actualité récente liée à la crise économique et financière soulève d'autres questions d'importance sur ses conséquences en matière patrimoniale et sur les comportements d'épargne des Français. Comment les individus gèrent-ils leur portefeuille financier face aux aléas boursiers caractérisés par une chute du CAC 40 de $40 \%$ et des cotations très erratiques en fin d'année 2008 ? Comment réagissent-ils dans leurs décisions immobilières après le durcissement des conditions de crédit et les perspectives de baisse des prix des logements ? Les attitudes vis-àvis du risque, la longueur de l'horizon décisionnel, la propension à transmettre à ses enfants se sont-elles modifiées?

Certaines études ont déjà mesuré les premiers impacts de la crise boursière et immobilière sur les patrimoines à partir des données de la comptabilité nationale. Si pendant la période d'avant la crise (2003-2006), le patrimoine net des ménages a crû à un rythme de plus de $10 \%$ par an, cette croissance s'est modéré en 2007, et la tendance devrait être à la baisse en 2008, une diminution de $3 \%$ étant prévue (Couleaud et Delamarre, 2009). Pour compléter cette analyse globale, il serait bon de disposer également de données microéconomiques.

Pour cela, l'enquête Patrimoine 2009 de l'Insee permettra de faire un premier constat : en comparant les patrimoines en 2004 et en 2009, on pourra déjà évaluer les conséquences de la chute des cours boursiers et de la baisse des prix immobiliers sur les avoirs des Français. La limite de cette analyse tient en ce qu'elle sera fondée sur des données en coupe instantanée qui n'autorisent pas un suivi des mêmes ménages avant et pendant la crise.

En revanche, des données longitudinales pourraient permettre d'analyser non seulement les mouvements patrimoniaux induits par la crise mais également les réactions plus subjectives des épargnants face au nouvel environnement économique et financier. En effet, la dynamique des préférences est un élément à prendre en compte dans les décisions individuelles. Par exemple, l'attitude des épargnants vis-à-vis du risque est-elle restée la même en cette période d'incertitudes ou a-t-elle évolué en fonction des évènements?

Parallèlement à l'enquête Patrimoine, il a donc été envisagé de réaliser en 2009, conjointement avec TNS-Sofres, une enquête auprès des épargnants français qui avait déjà été interviewés par le même institut en 2007 (voire en 2002 pour certains d'entre eux). La crise étant intervenue dans l'intervalle, on dispose là de l'opportunité d'une « expérience naturelle » pour étudier les changements de comportements des ménages français. Sans parler d'un véritable Panel, nous 
disposerons néanmoins d'échantillons d'individus pour lesquels nous aurons deux, voire trois points d'observations. Comme dans les enquetes de l'Insee, l'information recueillie dans ce « panel » concerne principalement la description sociodémographique du ménage, le montant, la composition et le mode de gestion de son patrimoine, le recensement de ses revenus, l'existence de transferts intergénérationnels reçus ou versés... Mais surtout, tout un questionnaire qualitatif vise à mesurer ses préférences en matière d'épargne (aversion au risque, préférence pour le présent, altruisme, etc.) (22).

On pourra de la sorte analyser l'impact de la crise en suivant les évolutions parallèles des patrimoines (montant et composition) et des préférences des épargnants - qu'il s'agisse de leur propension à prendre des risques ou de leur disposition à se projeter dans l'avenir.

\section{Luc Arrondel (Cnrs-PSE et Banque de France-DEMS-SAMIC)}

André Masson (Cnrs-PSE et Ehess)

Daniel Verger (Insee)

Les auteurs tiennent à remercier Stéfan Lollivier pour sa lecture attentive et ses remarques avisées. Pour cette étude, Luc Arrondel et André Masson ont bénéficié du soutien de la chaire "Risques et Chances de la transition démographique " (Fondation du risque).

\section{BIBLIOGRAPHIE}

Arrondel L. et Grange C. (2006), «Transmission and Inequality of Wealth : an Empirical Study of Wealth Mobility from 1800 to 1938 in France », Journal of Economic Inequality, 2006, n4, pp. 209-232.

Arrondel L., Guillaumat-Taillet F. et Verger D. (1996), « Montants du patrimoine et des actifs : qualité et représentativité des déclarations des ménages », Économie et Statistique, n 296-297, pp. 145-164.

Arrondel L. et Masson A. (2006), « Altruism, Exchange or Indirect Reciprocity : What Do the Data on Family Transfers Show? », in The Economics of Giving, Reciprocity and Altruism, Mercier-Ythier J. et S. C. Kolm (eds.), North Holland, chapitre 14, pp. 971-1053.

Arrondel L. et Masson A. (2007a), « Solidarités publiques et familiales », dans Une jeunesse difficile : Portrait économique et social de la jeunesse française, Cohen D. (éd.), Presses de l'ENS, Paris, chapitre 3, pp. 107-190.

Arrondel L. et Masson A. (2007b), Inégalités Patrimoniales et Choix Individuels, Economica, Paris.

Arrondel L. et Masson A. (2009), « L'efficacité économique peut-elle justifier l'augmentation des droits de succession? ", à paraître dans La question de l'intergénérationnel, sous la direction de A. Quéniart et R. Hurtubise, Éditions EHESP, Collection « Lien social et Politiques ».

Arrondel L., Masson A. et Verger D. (2004), « Préférences de l'épargnant et accumulation patrimoniale », Économie et Statistique, $\mathrm{n}^{\circ}$ 374-375.

22. Arrondel et al. (2005) montrent par ailleurs tout l'intérêt qu'il y a à classer les épargnants français dans quatre catégories en croisant leurs préférences vis-à-vis du risque et du temps. Selon cette typologie, les "bons pères de familles » (peu d'appétence pour le risque, horizon décisionnel de long terme) privilégient les actifs de précaution et de préparation de la retraite. À l'inverse, les " têtes brûlées " (goût du risque prononcé, préférence pour le présent) s'orientent vers les actions et les produits financiers liquides. Les « entreprenants " (peu averses au risque, vision à long terme) s'engagent dans des projets d'investissement incertains et de long terme. Enfin, pour les autres, "cigales prudentes » (peu d'appétence pour le risque, gestion au jour le jour), les stratégies patrimoniales sont centrées autour des encaisses de précaution à moyen terme. 
Arrondel L., Masson A. et Verger D. (2004), « Préférences face au risque et à l'avenir : types d'épargnants », Revue Économique, 2005, n 56, vol. 2, pp. 393-416.

Barthez A. et Laferrère, A. (1996), « Contrats de mariage et régimes matrimoniaux », Économie et Statistique, $\mathrm{n}^{\circ}$ 296-297, pp. 127-144.

Becker G.S. et Tomes N. (1986), « Human Capital and the Rise and Fall of Families », Journal of Labor Economics, ${ }^{\circ} 4$, part 2, S1-S39.

Becker G.S. (1991), A Treatise on the Family, Harvard University Press.

Bertaud G. et Malinvaud E. (1953), Enquête sur les dépenses d'habillement des Français en 1953.

Boissinot J. et Friez A. (2006), « Épargne et patrimoine des ménages : données macroéconomiques et données d'enquête », L'économie française, Insee Références, pp. 99-135.

Christelis D., Jappelli T. et Padula M. (2005), « Wealth and Portfolio Composition » in Börsch-Supan, A., Brugiavini A., Jürges H., Mackenbach J., Siegrist J., and Weber G. (eds.), Health, Ageing and Retirement in Europe - First Results from the Survey of Health, Ageing and Retirement in Europe. Mannheim : Mannheim Research Institute for the Economics of Aging (MEA), pp. 310-317.

Chauvel L. (1998), Le Destin des générations. Structure sociale et cohortes en France au XXe siècle, Presses universitaires de France, Paris.

Cohen V. et Demmer C. (2004), « Enquête sur le patrimoine des ménages : synthèse des entretiens monographiques », Document de travail Insee, ${ }^{\circ} 0401$.

Cordier M., Houdré C. et Rougerie C. (2006), «Les inégalités de patrimoine des ménages entre 1992 et 2004 », Les revenus et le patrimoine des ménages, Insee-Références, pp. 47-58.

Cordier M. et Rougerie C. (2004), « Patrimoine des ménages début 2004 — Le déploiement de l'épargne salariale », Insee Première, $\mathrm{n}^{\circ} 985$.

Cordier M., Houdré C. et Ruiz A. (2007), « Transferts intergénérationnels entre vifs : aides et donations », Insee Première, $\mathrm{n}^{\circ} 1127$.

Couleaud N. et Delamarre F. (2009), « Le patrimoine économique national de 1978 à $2007: 30$ années au rythme des plus-values immobilières et boursières », Insee Première, n ${ }^{\circ} 1229$.

Darmon C. et Pagenelle H. (2005), « Patrimoine : quand les ménages prennent de l'assurance », Insee Première, $\mathrm{n}^{\circ} 1015$.

Echevin D. et Parent A. (2002), « Les indicateurs de polarisation et leur application à la France », Économie et Prévision, $\mathrm{n}^{\circ} 155$, pp. 12-30.

Girardot P. et Marionnet D. (2007), « La composition du patrimoine des ménages entre 1997 et 2003 », France, portrait social, Insee-Références, pp. 155-174.

Guiso L., Japelli T. et Haliassos M. (2003), Stockholding in Europe, New York : Palgrave Macmillan.

Houdré C., (2007), « L'endettement des ménages début 2004 », Insee Première, n ${ }^{\circ} 1131$.

Kotlikoff L. J. (1992), Generational Accounting : Knowing Who Pays, and When, for What We Spend, The Free Press, New York.

Landais C. (2007), « Les hauts revenus en France (1998-2006) : une explosion des inégalités ? », Mimeo, Paris School of Economics.

Le Bris D. et Hautcoeur P.C. (2008), « Challenge to Triumphant Optimists ? A New Index for the Paris Stock-Exchange (1854-2007)», Mimeo. 
Lollivier S. (2005), « L'Insee et les enquêtes sur les patrimoines », Économie et Statistique, n ${ }^{\circ} 374-375$, pp. 3-7.

Masson A. (2009), Des liens et des transferts entre générations, à paraître aux éditions de l'Ehess, Paris.

Masson A. et Pestieau P. (1991), « Types et modèles d'héritages et leurs implications », Économie et Prévision, $\mathrm{n}^{\circ} 100-101$, pp. 31-72.

Masson A. et Verger D. (1996), « Le patrimoine des Français : comportements et disparités », Économe et Statistique, n ${ }^{\circ} 296-297$, pp. 3-11.

Merton, R.C. (1971), « Optimal Consumption and Portfolio Rules in a Continuous Time Model », Journal of Economic Theory, 3, pp. 373-413.

Modigliani F. et Brumberg R. (1954), « Utility Analysis and the Consumption Function : an Interpretation of Cross-Section Data », in Post-Keynesian Economics, pp. 388-436, K. K. Kurihara (ed.), Georges Allen and Unwin, Londres.

Pinçon M. et Pinçon-Charlot M. (1996), Grandes fortunes, Dynasties familiales et formes de richesse en France, Payot, Paris.

Piketty T., (2001), Les hauts revenus en France au XXe siècle. Inégalités et redistributions 1901-1998, Grasset, Paris.

Piketty T., Postel-Vinay G. et Rosenthal J.L. (2006), « Wealth Concentration in a Developing Economy : Paris and France, 1807-1994 », American Economic Review, vol. 96, n 1, pp. 236-256.

Tanay A. (2002), « Les actions plus rémunératrices que les obligations et l'or au $\mathrm{XX}^{\mathrm{e}}$ siècle », Insee Première, n ${ }^{\circ} 827$. 


\section{La Collection Insee-Références}

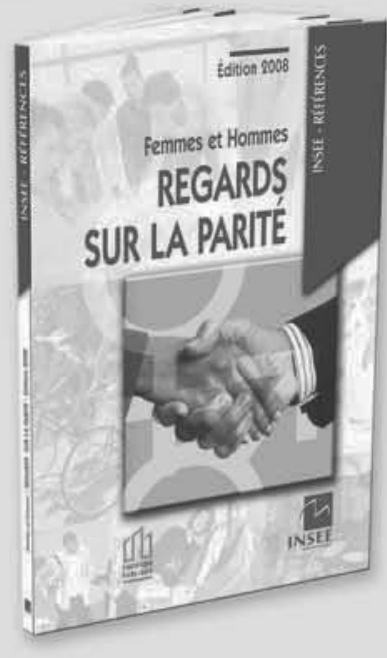

$>$ L'ouvrage $15 €$

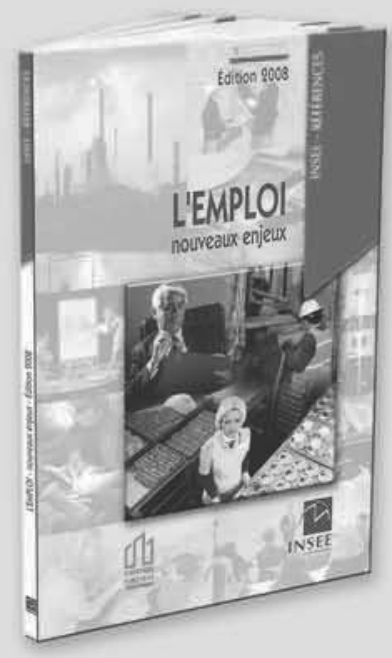

$>$ L'ouvrage $16,50 €$

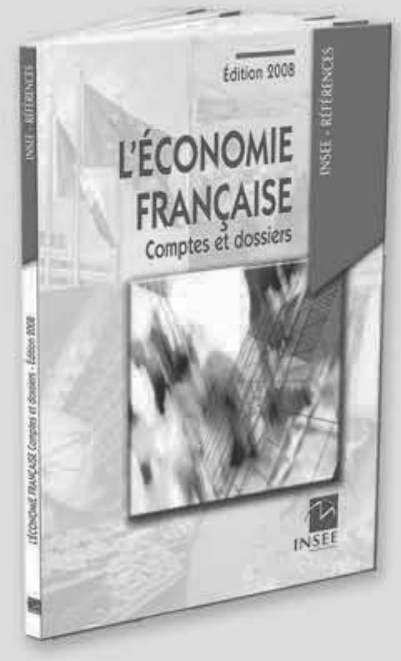

$>$ L'ouvrage $15 €$

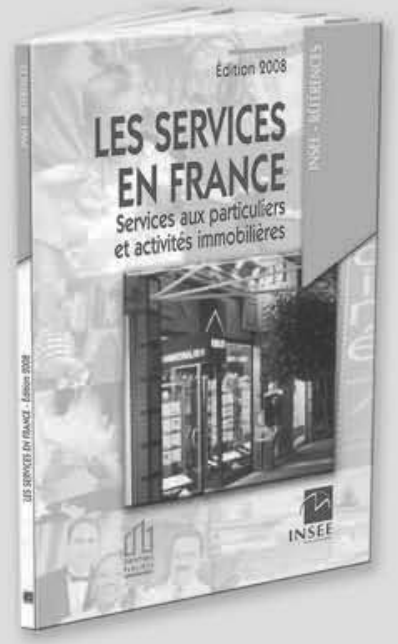

$>$ L'ouvrage $16,50 €$

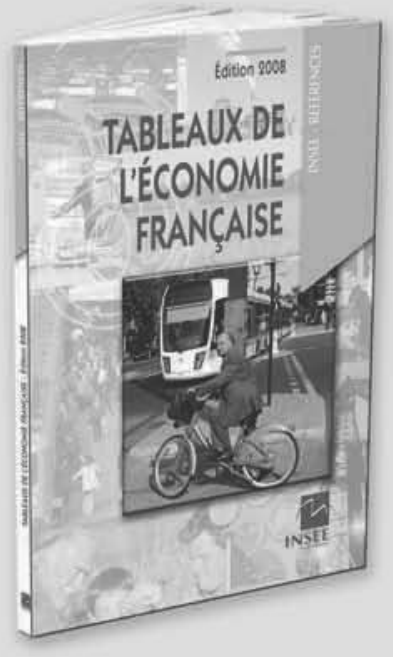

$>$ L'ouvrage $16,50 €$

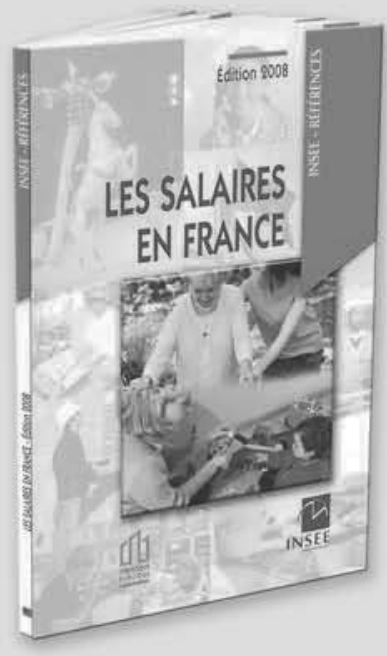

$>$ L'ouvrage $16,50 €$

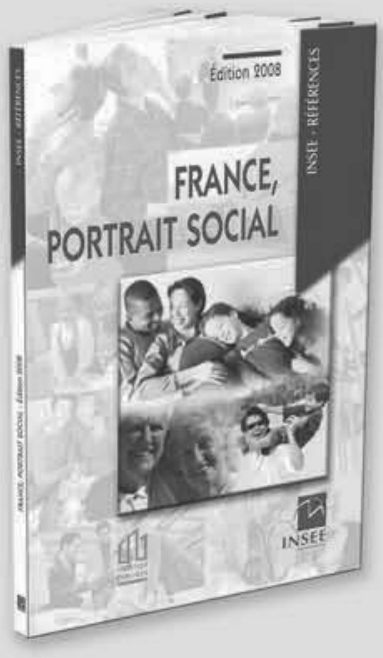

$>$ L'ouvrage $16,50 €$

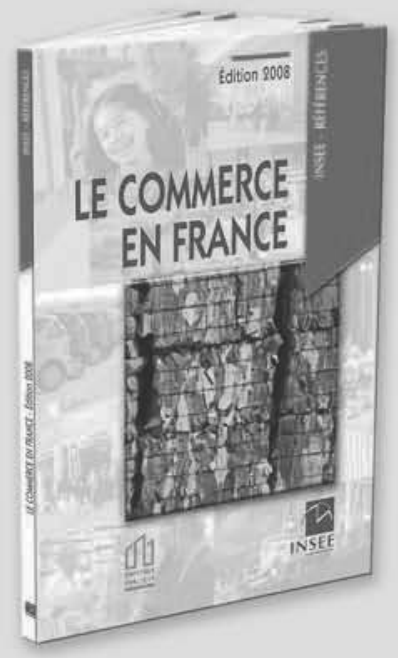

$>$ L'ouvrase $16,50 €$

Prochainement : > L'industrie en France - édition 2008

En vente en librairie, par correspondance et sur www.insee.fr

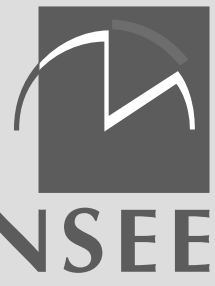

